Journal of Contemporary Educational Research

Research Article

\title{
An Analysis on the Teaching Reform of the Basic Course of Zhuangyi Undergraduate Major
}

Qinghuai Zhang, Hui Gao(Co-first author), Gang Fang, An Huang, Luqiu Wei, Xiusong Tang, Qiuxia Chen, Yuying Lan*, Yuzhou Pang*

Guangxi University of Traditional Chinese Medicine, Nanning 530200, Guangxi Province, China

Funding: Guangxi Higher Education Undergraduate Teaching Reform Project (No.2018JGZ124); Teaching Reform and Research Project of Guangxi University of Traditional Chinese Medicine(No.2018C59).

\begin{abstract}
The basic course of Zhuangyi is a part of the undergraduate course which teaching content covers the basic theory of Zhuangyi, diagnosis of Zhuangyi, basic theory of traditional Chinese medicine, diagnostics of traditional Chinese medicine, anatomy of Western medicine and pathology. Whether we can lay a solid foundation for the professional knowledge of Zhuangyi is related to the ability of students to enter clinical posts. This paper explores the teaching method reform of basic courses of Zhuangyi, in order to cultivate high-level talents more suitable for the current social requirements.
\end{abstract}

Key word: Training of post competency; Foundation of Zhuangyi; Teaching methods; Reform

Publication date: August, 2020

Publication online: 31 August, 2020

*Corresponding author: Yuying Lan,

yuyinglan2005@126.com; Yuzhou Pang,pangyz422@ sina.com

With the development of medical higher education, the limitations of traditional teaching mode have become increasingly prominent. The innovation and development of medical teaching mode has become the focus and difficulty of current medical higher education teaching reform ${ }^{[1]}$. There are more and more new teaching methods such as BPL (problem-based teaching method), CPL (case-based teaching method), TBL (team oriented teaching method) and so on, which provide new choices for medical education methods. As a new medical specialty, ethnic medicine is still in continuous exploration of talent training mode. There is a lack of innovation in specialty setting or curriculum system.
How to speed up the pace and cultivate Zhuangyi talents more suitable for the needs of modern society is an urgent problem to be solved. At present, the teaching method of basic course of Zhuangyi is obsolete and the teaching method is single. It is particularly urgent to explore how to reform the teaching method of Zhuangyi course and cultivate high-level talents needed by the society.

\section{The reasons for the reform of basic courses of Zhuangyi}

\subsection{The information age puts forward higher requirements for the teaching of basic courses of Zhuangyi}

In today's society, the information revolution has impacted almost all fields. With the advent of the information age and the rise of online courses, the role of teachers in the classroom will no longer be indoctrinated teaching mode, and the classroom will no longer be limited to fixed classrooms and class hours. We should make full use of scientific and technological information means to improve the teaching effect, so as to avoid the phenomenon of low enthusiasm of students in the course. Teaching with mobile phones and tablet computers in coastal or first tier cities is no longer a new means. Therefore, scientific and technological teaching is of great significance to solve the problems of large class system, collective system and flood irrigation in China. This requires that some older teachers in colleges and universities must be familiar with modern teaching tools, combine online and offline, strive to get rid of textbooks, diversify knowledge carriers, vividly convey 
knowledge, and explore the use of "rain class", "micro video" and "Moodle network course platform" for teaching [2-4]. At the same time, in the information age, there are various ways of knowledge transmission and rapid update and development. Therefore, the teaching of basic courses must not be conformed to the rules, and all chapters and plates are the same. We should actively supplement the new progress of basic knowledge research.

\subsection{The post demand puts forward higher requirements for the teaching of basic courses of Zhuangyi}

The 13th five year plan of the state emphasizes that in order to deepen the reform of vocational education mode, talents need to be based on posts and take applicable post requirements as standards ${ }^{[5]}$. The basic knowledge of Zhuangyi has certain abstractness, such as Yin and Yang noumenon, three Qi synchronization. It is not easy to master it in single words. Therefore, students lack certain initiative and enthusiasm in learning and mastering basic knowledge and content ${ }^{[6]}$. In the teaching process of basic courses of Zhuangyi specialty, we should take "quality target", "knowledge target" and "skill target" as reference requirements. In the actual teaching process, the teaching process of some Zhuangyi knowledge is slightly simple, the proportion of theoretical teaching is too large, and the proportion of practical teaching is too small. However, medicine is a specialty based on theory and practice first, and theory guides practice and practice testing theory, students can not only test the mastery of theoretical knowledge in practice, but also make teachers and students think about the truth of the theory, which is of great significance to feed back the theory, promote its further development and keep pace with the times. Basic knowledge is an important foundation for students to engage in clinical work, and it is also an important theoretical basis for students to construct clinical thinking mode, form clinical prescriptions, display treatment methods and guide rehabilitation process. Therefore, the teaching of basic courses must be aimed at clinical practice, and the post requirements must have the dual background and skills of theory and practice at the same time. Such teaching can not only avoid the boring theory, but also enrich the teaching content, improve the students' interest and attention, and promote the long-term sustainable development and progress of "teaching learning medicine". The basic course of Zhuangyi not only requires students to have good basic theoretical knowledge of Zhuangyi, but also requires students to have strong practical ability. Only by strengthening the combination of theory and practice can we cultivate practical clinical talents of Zhuangyi.

\subsection{The inheritance and development of ethnic medicine put forward higher requirements for the teaching of basic courses of Zhuangyi}

The inheritance and development of ethnic medicine must take the inheritance of basic theory as the premise, guide clinical practice with theory, and take senior talents with solid theoretical basic knowledge as the leader. Zhuangyi is one of the important methods for the prevention and treatment of diseases in Zhuang nationality area through long-term social practice. With the continuous development of Zhuangyi, how to better carry forward and develop Zhuangyi culture has brought us challenges and opportunities. Guangxi University of traditional Chinese medicine is the only educational base for cultivating high-level Zhuangyi Talents in China, and it also shoulders the mission of inheriting the excellent culture of Zhuang nationality.In the early stage, after decades of literature mining and on-thespot investigation of Zhuang nationality inhabited areas, Zhuangyi culture, theory, clinical technology and folk characteristic therapy have been comprehensively summarized, studied and developed[7-9]. On the basis of further improvement and excavation, we should attach importance to the platform of colleges and universities, deeply carry out the education of basic courses of Zhuangyi, so that the majority of students who are really interested in Zhuangyi will strive to master the essence of Zhuangyi culture and integrate the basic theory of Zhuangyi. Therefore, there are higher requirements for the teaching of basic courses of Zhuangyi, especially for the construction, configuration and teaching methods of basic courses of Zhuangyi. We should have a broader idea of academic research and development based on theory, and strive to realize the education of scientific research and academic feedback. The contention of a hundred schools of thought is more conducive to the improvement and teaching of basic curriculum knowledge in Colleges and universities Broadcast $^{[10]}$.

\section{Teaching reform of basic courses in Zhuangyi specialty}

\subsection{Building a reasonable curriculum system}

The basic curriculum of Zhuangyi pays attention to the coordinated development of students' knowledge, 
ability and quality. The curriculum system based on the examination content structure of Zhuangyi practitioners will be further sorted out. Only by combining the requirements of Zhuangyi practitioners, can the principle of "use as soon as possible" be realized.Through the establishment of a simplified and focused teaching system, remove miscellaneous courses, focus on important courses, simplify the teaching structure, and pay attention to return to the professional knowledge.The author believes that the reasonable curriculum system of ethnic medicine should be combined with Zhuangyi, traditional Chinese medicine and Western medicine, focusing on the core task of guiding the clinical optimistic disease.It can be explored from the following three aspects:First, it is necessary to form a teaching system based on traditional Chinese medicine course, Zhuangyi basic course as the key and characteristic, and Western medicine course as supplement.At the same time, it is necessary to form a traditional culture teaching atmosphere of Zhuangyi, and make it more clinical in the process of theoretical teaching, so that "Zhuangyi Culture Basic Theory Zhuangyi Diagnostics clinical practice" will be integrated and developed, and a new cross teaching mode of theory and practice will be formed.Secondly, it is necessary to cultivate Zhuangyi base, especially to let students participate in the cultivation and management of Zhuangyi in person, from passive acceptance to subjective initiative, so that Zhuang pharmacy and Zhuangyi prescription science can be combined, cross teaching, and more field teaching should be carried out, so that students can go out of the classroom, release vitality, explore the image and concrete of prescriptions, and form a curriculum system combining relevant courses and giving consideration to both indoor and outdoor.The third is to strengthen the clinical thinking and core ideas of Zhuangyi as the main line, such as Yin and Yang as the basis, three Qi synchronization, three channels and two routes, theory of toxin deficiency and pathogenicity, etc. the core theory of Zhuangyi must be deeply rooted in the hearts of the people. In the teaching process, the diagnosis and treatment technology of ethnic medicine and the simple, effective and economical external treatment method of Zhuangyi should be highlighted, and its positive effects in public health, disease prevention and late rehabilitation should be analyzed The function is to let every student be both the receiver and the disseminator, so as to achieve the teaching purpose of learning culture, consolidating the foundation, being able to practice and promoting the inheritance.

\subsection{Strengthen the "three basic" training of Zhuangyi specialty}

The cultivation of medical talents is carried out around the "Three Basics" (basic theory, basic knowledge and basic skills). Among them, basic theory teaching is the foundation and foundation. At this time, students have just entered the door of medicine and have no medical knowledge and experience. How to combine theoretical teaching with practical operation needs to study hard. The ultimate goal of basic teaching is to lay a solid foundation, that is to try every means to make these medical theories deeply imprinted in students' minds, which is the key problem to be solved. Many of the ancient Chinese medicine studies are children's skills, that is, whether you understand or not, first recite the classics, soup and other basic things. After you learn the clinical practice, these basic things will flow out of your mind and guide your clinical practice. Therefore, the key to the teaching of basic courses is to let students write down the theory, and how to make students love learning and remember these theories is what teachers need to do.

\subsection{Innovative and diversified teaching methods and modes}

Nowadays, the traditional and single teaching mode can not bring the significance and value of basic courses of Zhuangyi into play, and the teaching goal of cultivating students' post competency is also difficult to achieve. In the context of advanced information technology, to give full play to the role of modern network, computer and multimedia and other advanced technology, teachers must reshape their own role, take students as the center, break the sense of teaching space-time boundary, adopt mixed teaching mode, and replace rigid set and collective indoctrination with personalized learning ${ }^{[11]}$. The problem-based teaching method, casebased teaching method and team oriented teaching method are introduced into the medical education classroom, which makes MOOC, micro class and other mobile phone APP modes and animation really enter the learning life. Cultivate students' open thinking and ability to solve problems independently. In the basic courses of Zhuangyi specialty, theoretical teaching and practical operation can be combined organically. In the theoretical teaching class, operation demonstration or practice can be appropriately inserted, which can not only eliminate the dullness of teaching, but also increase the teaching hours of clinical operation. For example, 40 class hours of basic theory teaching and 40 class hours of diagnosis of Zhuangyi can be 
taught together Clinical field teaching, with theory to guide practice, real cases to demonstrate the diagnosis process. Another example is Zhuangyi prescription science should be combined with teaching, what kind of medicine is in the prescription, at this time, the main help of the medicine and its efficacy are explained, so as to realize the combination of medicine and prescription. To guide the cultivation of interdisciplinary talents, reduce the theoretical learning burden of students, and conduct more field activities. Grow up and become talents through "participation". At the same time, in the teaching process, teachers and students can exchange roles, organize students to make PPT and give lectures on stage, so as to realize the interaction between teaching and learning ${ }^{[12]}$. So as to establish an effective operation mechanism of the combination of practical teaching and practice.Focusing on the post competency of students, we should focus on cultivating students' professional quality, professional thinking ability and production practice ability. We should integrate the knowledge of Zhuangyi Practitioners' examination points into the normal teaching, so that students can establish the concept of early contact with clinical practice and practice repeatedly in clinical practice as soon as possible, and carry out more discussion type exchanges, so as to cultivate students' enthusiasm and initiative, so that students can master the theory of Zhuangyi in time. Taking the cultivation of post competency as the core, the classroom teaching and clinical practice are organically connected, which runs through the course learning of Zhuangyi.

\section{Conclusion}

To sum up, the teaching content of Zhuangyi major covers three medical systems: Zhuangyi, traditional Chinese medicine and Western medicine. Whether we can learn the basic courses of Zhuangyi is very important for us to use theory to guide clinical practice and master clinical skills. Only by mastering the basic courses of Zhuangyi can we have a firm plan and better guide the clinical practice of Zhuangyi. We should construct the curriculum system of Zhuangyi reasonably, flexibly apply innovative teaching methods and methods, and strengthen the basic skills of basic courses of Zhuangyi students, so as to be better qualified for clinical work.

\section{Reference}

[1] Wei JB, Lan Z, Liao XL. Six trends of medical teaching mode reform[J]. Shezhi, 2017, 4: 544-547.

[2] He XW. Research on the application of information technology in medical education and Teaching $[\mathrm{J}]$. Information and computer (theoretical Edition), 2019, 6: 238-239.

[3] Chen W, Li L, Zhao XJ, et al. Teaching reform practice of histology and Embryology based on "rain class" course[J]. Chinese Journal of histochemistry and cytochemistry, 2019, 28(6): 560-563.

[4] Li P, Wang HQ, Dai YX. Research on the practice of hybrid teaching mode based on Moodle network course platform in basic medical course teaching[J]. Henan Education (Higher Education), 2019(12): 116-118.

[5] Ma CY. Exploration and practice of project-based curriculum reform oriented by professional post competency[J]. Computer knowledge and technology, 2018, 34: 159.

[6] Lin JY, Pang YZ, Zhang QH, et al. Discussion on the cultivation of humanistic quality in Zhuangyi teaching [J]. Asia Pacific traditional medicine, 2017, 18: 166-167.

[7] Lan YY, Qin LL, Zhang QH, et al. Protection of Zhuangyi culture inheritors and preliminary study of Zhuangyi culture transmission[J]. Journal of Guangxi University of traditional Chinese medicine, 2019, 1: 132-135.

[8] Zhang YS, Duan XL, Pang YZ, et al. The practice and discussion of introducing Zhuangyi Humanities and culture curriculum into Zhuangyi Undergraduate Specialty[J]. Comparative study on cultural innovation, 2019, 3(4): 142-143.

[9] Liang XL, Liu JW, Tang HZ, et al. Preliminary study on the promotion of Zhuang Traditional Medicine Culture[J]. China National Expo, 2018(7): 4-6.

[10] Tan J, Cai RL, Lu X, et al. Application of teaching and research community consciousness in histology Experiment Teaching $[\mathrm{J}]$. Modern medicine and health, 2020, 36(8): 1250-1252.

[11] Liu QY, Liu XY. Informatization and medical teaching reform[J]. Chinese PLA Journal of Hospital Management, 2019, 26(2): 183-187.

[12] Liu PA, Ding YY, He L, et al. The relationship between innovation and application-oriented medical students[J]. Research on Integrated Chinese and Western medicine, 2016, 8(3): 162164. 